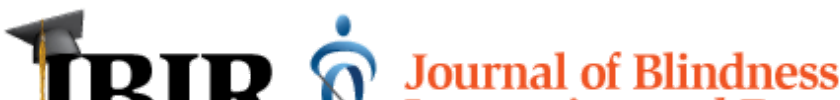

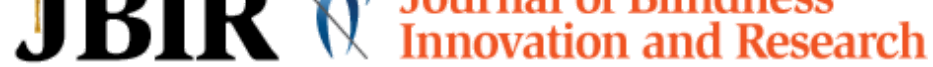

Home $>$ Vol 7, No $1(2017)$

\section{Blind Adults' Perspectives on Technical Problems and Solutions When Using Technology}

\author{
By Anne Jarry, Claude Chapdelaine, Sri Kurniawan, and Walter Wittich
}

Anne Jarry is an associate professor in the VRT graduate program at École d'optométrie, Université de Montréal.

Claude Chapdelaine is a senior advisor on the Vision and Imaging Team at the Centre de Recherche de Montréal (CRIM), Canada.

Sri Kurniawan is a professor in the Computational Media and Computer Engineering departments at the University of California Santa Cruz Baskin School of Engineering.

Walter Wittich is an assistant professor at École d'optométrie, Université de Montréal.

\begin{abstract}
This study investigated the technical difficulties and technical assistance that blind adults experienced when interacting with computers and computing applications.

Methods: The study utilized surveys in French and English, delivered either online or as emailed Microsoft Word documents. The questions focused on the frequency, nature, and causes of issues.

Results: Fifty-six people using sight substitution technology participated in the survey. Regarding types of problems experienced, 66\% were solved without help from sighted people. Of those that were unsolvable without sighted help, differences were found between English-speaking Americans and French Canadians. French Canadian respondents mostly indicated usability issues (65\%). Discussion and Conclusion: The findings indicate that the most cited technical problems related to upgrading or switching to new operating systems and inaccessible content.
\end{abstract}

\section{Keywords}

Blind adults, technology, accessibility, professional development

It has been claimed that computers and the Internet are two of the most significant developments since the invention of braille, as for the first time ever many blind and partially sighted people have access to the same wealth of information as sighted people and on the same terms (Abner \& Lahm, 2002). Computing technology is potentially a democratizing tool, promising greater accessibility to information, services, and society. While this statement is generally true, it is important to systematically investigate the perceptions of those with non-functional vision to ascertain whether current computing technology is accessible and truly functional for independent use by this user group, or whether this group has to rely on sighted/technical help to use such technology. This issue is not a simple matter, as those specialising in technology instruction and those providing technical support for blind users are struggling to stay informed and are often not able to keep pace with this rapid development (Lazar, 2009). Furthermore, a lack of information sharing and collaboration has been identified by researchers, service providers, developers, and users of assistive technology (Percival, 2012).

Additionally, the training provided to professionals in assistive technology has been found to be insufficient to meet the growing needs of blind users (Smith, Kelly, \& Kapperman, 2011; Zhou, Parker, Smith, \& Griffin-Shirley, 2011).

There are still accessibility and usability issues that pose major challenges, such as poor accessibility on the Internet, lack of spoken feedback, cost, rapid changes, time required to learn new devices, new keyboard commands and gestures, etc. As Petty stated in 2005, "The barriers do exist-they're all too real-and few people with limited vision, or no vision, have even begun to work virtually. For most, virtual work just isn't 'on the radar screen'" (p. 8).

To address this crucial steppingstone to social inclusion, a comprehensive research program is required. Such a program should include the needs of the users, the concerns of the clinicians providing training in assistive technology, and the perspectives of the technicians involved in technology development. More frequently, companies and service providers include the end-user in the initiation, development, and delivery of their products, while governments and organisations are increasingly looking to users to help set standards (Bastien, 2015).

The goal of our study was to help practitioners, vendors, creators of technology, and graduate students hear the voices of blind adult computer users in order to gain a better understanding of the difficulties and solutions that they experience when using various kinds of computing technology. For this paper, the goal was to represent the voices of functionally blind English-speaking Americans and French-speaking Canadians regarding their use and level of challenge when interacting with computer technology. 


\section{Methodology}

To gain a better understanding of these types of problems an online survey was created. The survey was created in both French and English and consisted of 25 questions, which were divided into six main categories: 1) demographics; 2) type of technology used; 3) duration of use; 4) ease of use; 5) occurrences of technical issues, their frequency, circumstances and consequences; and 6) occurrences of difficulties resulting in the need to ask for assistance. This mixed method survey required answers that were either quantitative (e.g., duration of use), yes or no response (e.g., use of a mobile device), or open-ended short answer questions that required a written response (e.g., If you experienced switching operating system or upgrades, please describe your experience when you started using the new operating system). As two languages were used for the survey, one of the co-authors translated the French responses and merged the data. These written responses were then compiled and coded by major themes, based on specific words that were used in the answers. All responses were taken into account, even if the questions were not fully completed. In addition, participants could choose multiple answers for questions such as types of device used, where to ask for help, etc.

\section{Materials}

Samples of the questions asked within the six main sections of the survey are presented below.

1. Demographics: In this section close-ended questions were asked about our participants, including age, gender, level of blindness, and years of blindness. Ex. Please choose which best describes your level of blindness.

2. Type of technology used: Participants were asked, using close-ended questions, the type of operating system used. Ex. Please write the type of operating system used (select all that apply): Windows, Mac, iOS, Android, I don't know, Other (please specify).

3. Duration of use: This section was intended to gain, through open-ended questions, the number of years of using different types of operating systems and mobile devices. Ex. Please write the number of years you have been using a tablet (e.g., iPad or Google Nexus) (Answer 0 if you never use it).

They were also asked in open-ended questions if they had experienced switching operating systems. Ex. Did you experience firsthand switching from one operating system to another (i.e. Windows XP to Windows 7 or from Windows to Mac), describe the change, and from what to what.

4. Ease of use: Close-ended and open-ended questions were asked to identify their prefered operating system. A list of multiple choices were given to identify which operating system the participants used most often. And then the following open-ended questions were asked: Which one device do you feel is the most easy to use? Why do you feel that this device is the easiest to use? For the device you used most often, how did you get trained to use it?

5. Occurrences of technical issues, their frequency, circumstances and consequences: In this section questions were geared at elucidating through open and close-ended questions the methods used to solve issues. Ex. Please describe 3 examples of problems you solved without assistance. When/if you experience technical problem you cannot solve yourself, who are the three persons or organizations you contact for help (in the order of your preference)?

6. Occurrences of difficulties resulting in the need to ask for assistance: Questions were geared at determining, through open and close-ended questions, the types and level of difficulties encountered and the types of solutions initiated. Ex.Can you give a gross estimate (in percentage) of the problems you can solve yourself and those you cannot solve because it requires a sighted person? Think of your most recent technical problem that was solved with sighted assistance and the way it was solved. If that problem occurs again, can you solve it yourself? If the person you contacted for help is a friend or relative, when do you feel it's OK or not OK to ask for help? Do you feel you get technical help in a timely manner? Please explain. Do you feel you get accurate technical help? Please explain. If you answered "no" to either of the two preceding questions, do you feel this situation discouraged you from using technology?

\section{Participants}

To participate, the respondents needed to, be over 18 years old, have no remaining functional vision, make use of a screen reader, and regularly use a computer (laptop or desktop) and/or mobile device.

The survey link or the attachment was sent by email to multiple organizations working with visually impaired adults. In Quebec, Canada, these included the user committee of the Institut Nazareth et Louis-Braille Rehabilitation center, the Quebec Blind Sports Association (I'Association sportive des aveugles du Québec), and the Association of Blind and Amblyopic Individuals of Québec (Regroupement des aveugles et amblyopes du Québec). The American participants were recruited from a database of blind adults who had participated in other studies and consented to being contacted for future research at the University of California, Santa Cruz (UCSC). Institutional Review Board (IRB) approval was secured independently in the US through UCSC and in Canada through the Centre de Recherche en Informatique de Montréal (CRIM).

Procedure. The directors of the various organizations were contacted to obtain permission to send an email invitation to all their members. The email included a message explaining the goal of the research project, as well as detailed instructions on how to complete and send back the survey. As an incentive to complete the survey, participants were entered into a prize drawing to win a $\$ 50$ Amazon gift card. Due to technical difficulties with the accessibility of the survey, the dissemination of the English and French versions differed. The English version was made available through Survey Monkey (www.surveymonkey.com) via a direct link in the email, whereas the French version was distributed by email as an attached Microsoft Word document.

Data collection ran from October 2013 to January 2014. All data was pooled into a Microsoft Excel spreadsheet and then coded for content analysis. The French Canadian responses were translated and merged with the English American data.

The survey was completed by 43 English-speaking American and 19 French Canadian respondents, yielding a total of 62 returned questionnaires. Of the 62 participants, six who used low vision access technology instead of screen readers were excluded from the analysis. Of the 56 remaining participants, 40 English-speaking Americans took the survey online and 16 French Canadians used the Word version of the questionnaire. 


\section{Results}

\section{Demographics, Technology Use, and Duration of Use}

A detailed quantitative description of the first three sections of the survey (demographics, technology use, and duration of use) is shown in Table 1. The number of male to female respondents was almost equivalent, and the majority were between the ages of 40 and 65 . When asked about upgrading or switching operating systems, fifty respondents indicated experience with upgrading or switching systems. On average, the respondents have been using desktops the longest (39 years), followed by laptops (27 years), then smart phones (4 years), and most recently tablets ( 0.5 year).

When asked about the type of system used, participants could choose multiple answers. All but 4 respondents have used or currently use Windows OS, 31 iOS, 15 Mac, 3 Linux, and 7 Android.

\section{Ease of Use}

When participants were asked which devices were easier to use, the majority listed devices with keyboards as the most popular. "Easy" because of experience (muscle memory) was mentioned 17 times and "better" because of efficiency in multitasking was mentioned 7 times.

This was supported by responder comments:

It has a physical keyboard and I can control where I am on the screen with the arrow keys. I can also use shortcuts and the screen reader can be manipulated more easily. I can have all eight fingers and both thumbs in place and don't have to hunt and peck for keys.

Also, ease with simple and similar gestures for navigation on mobile devices was mentioned 13 times.

The pie-chart in Figure 1 indicates that most respondents preferred working with desktop computers (20 respondents, 35.7\%) for ease of use, while preference for using laptops came in close second (19 respondents, 33.9\%), and iPhones in third place (10 respondents, $17.8 \%$ ). Respondents who indicated "Other" as a preference referred to products such as braille devices, talking book recorders, etc. No participants indicated a preference for using a tablet. One participant mentionned that the prefered tool depended on the purpose of the task:

It depends upon the purpose. For skimming email or social media, the iPhone is usually my tool of choice. This is because the interface is simple and can be handled rapidly. For other tasks such as composition of a complex documents, using a small device and Pages can be a nuisance, so my desktop usually is the tool of choice.

\section{Occurrences of Technical Issues and Their Frequency, Circumstances, and Consequences}

The majority of challenges faced by participants arose at the moment of changing operating systems. Given the written feedback from the questionnaires, $41 \%$ of the experiences of participants with upgrading to new technology were labeled as negative. Participants mentioned a huge learning curve. Participants commented: "Horrible, I was obliged to get help. I would have liked to have training but at that time nothing was offered"; "Up until windows Vista...there was very little change in how I used my computer.Windows Vista was hard to learn and changing from Vista to Windows 8 was worse. Windows 8.1 is another change and not as easy to use with a screen reader as Windows 8.0"; and, "It took me a year to get used to the gestures required for Voiceover on the iPad and iPhone."

Fourteen percent of the reported experiences were identified as positive. Example comments included: "Generally fairly intuitive"; "It was nice to have similarities"; "Usually smooth except for switch from XP to Windows 7 and beginning use of iOS"; and "It was a bit hard to get used to at first, but honestly I think the change was way better."

A further $32 \%$ of responses were categorized as varying (i.e., a combination of positive and negative experiences in one response): "Confusing at first. My first switch was before Google, so I had to learn a lot by trial and error. I couldn't often call support if I got stuck because techs would often talk about clicking on things, or finding the blue bar at the top of the screen etc. and I hadn't learned to translate that into something I could use. Later conversions were easier because Google was more of a thing, as well as mailing lists for blind users"; "Steep learning curve"; and "bought a braille book from NBP [National Braille Press] that was helpful." Thirteen percent did not describe their experience.

\section{Occurrences of Difficulties Resulting in the Need to Ask for Assistance}

In the next section of the survey, participants were asked about the types of problems that they encountered and were able to solve on their own. Sixty-six percent of problems mentioned by these users were solved without help from sighted people. Almost threequarters of these common types of problems were categorized into four major types of problems: a) fixing screen reader issues, b) finding strategies when lack of feedback occurs, c) installing/updating, and c) finding functions or strategies in a program or on the Internet to complete a task. 
As for types of problems that the blind respondents were unable to solve by themselves, for English-speaking respondents, the most frequent problem was inaccessible content (e.g., CAPTCHA, improperly labeled buttons, links, and images). These problems composed $57 \%$ of the accessibility issues that could not be solved without outside help. In contrast, the French Canadian respondents mentioned usability issues (e.g., learning to navigate new applications, configuring new devices, and learning new keyboard commands) as the most frequent problems for which they needed help (65\%).

Furthermore, as shown in Table 2, 29 English respondents said that they did not seek help from a sighted person, but rather considered themselves to be self-taught or chose to access Internet searches or training tools. In this same language group, 11 mentioned receiving training from rehabilitation centers or from trainers working for assistive technology manufacturers. Of the sixteen French respondents, 13 mentioned seeking training or support through rehabilitation services.

Participants were also asked to describe up to three examples of problems they could solve by themselves, which included: resetting passwords, learning to navigate tricky but usable applications and programs, or converting files. In addition, problems relating to screen reading software not speaking were mentioned 49 times in the responses. Comments for solving problems by themselves included: "Use muscular memory to unmute a computer without sighted assistance"; "Trying complex webpages with different browsers (Explorer versus Firefox), or on the iPhone if the screen reader on the PC isn't cooperating well"; "Configuring email problem"; "Screen reader not speaking due to one reason or another because a program freezes or something. I just cheat and either force the program to shut down or restart my computer."

As for those situations requiring sighted assistance, problems included the inability to navigate on a website for grocery shopping, and inaccessible websites with visual-only CAPTCHA, flash, or lots of images. Other emerging problems included "graphical aspects of web sites not reachable via a keyboard," or "PDF forms that do not meet PDF/UA standards and cannot be completed with a screen reader."

Participants were also asked to identify the first three people/organizations that they approach when they need help and in which situations they did or did not feel comfortable asking for assistance. With regard to when they felt comfortable asking for help from friends/relatives, answers referred to the time of the day, how busy the friends/relatives were, and how time-consuming and complex the problem was. Comments included, "When the person is not too busy and if I am sure I cannot solve the problem myself and if I do not feel that I am being a bother and when I am about in tears," or "When I have tried everything I know, but still cannot accomplish the task. Especially when I'm pretty sure that the problem deals with accessibility." Some respondents stated that if they felt the problem would be too time-consuming, they would rather give up than bother their friends/relatives.

Finally, participants were asked to describe other issues related to computer accessibility and usability that they viewed as important. As shown in Appendix A, French respondents pointed out the importance of getting access to tutorials: "It would be nice to have tutorials or self-guided resources." Americans mentioned the numerous difficulties when speaking to technical support staff at large companies: "Telling a person who is blind where something is on the screen is pointless"; "They should be trained on the basics of screen reader use and function so they can communicate more effectively with blind customers," and "Reps should learn how to describe by key strokes the products they are supporting."

\section{Discussion}

The goal of this study was to explore the issues faced by blind users in the context of their current use of computer technology. As presented in Table 1, the age distribution showed that the respondents were mostly working-age adults, most had a visual impairment from birth, and most had gone through various upgrades and OS changes. Therefore, these were blind persons who were quite experienced in the use of computer technology. At the time of this research, desktops and laptops were found to be the easiest to use but we saw an increase in early adoption of mobile devices.

Even with this experienced sample, we observed that accessibility barriers and usability issues continue to cause major challenges that require blind users to seek outside help. This is especially important as the second most frequently mentioned problem related to a lack of feedback when something went wrong. Across languages, the problems related to accessibility and usability affected all of the respondents.

Interestingly, in this study, usability issues and the need for training support from rehabilitation professionals were more frequently cited by French-speaking Canadians than by English-speaking American computer users. This difference is possibly due to the fact that the keys are in different locations on English and French keyboards, adding the need for assistance when learning where the keys are located, or to change keyboard settings from English to French when starting with a new operating system. Also, the fact that there are limited commercially designed screen reading tutorials available in French, thereby reducing the possibility of learning through selftraining methods. Presently, commercially designed tutorials are only available in English with screen reading applications such as Jaws and Window-Eyes for OS systems. At the time that the data was collected for this study, Jaws in French was regrettably one version behind the English edition, causing compatibility problems with newer versions of programs such as Internet Explorer and Microsoft Office Suite.

The results highlight the ability of our participants to solve many issues without sighted assistance. These participants demonstrated real tenacity when solving problems and when needing to learn layouts and configurations for new applications or devices. However, this may only apply to moderate to experienced users and so may not adequately represent the overall population. Our findings reveal that a steep learning curve is often needed to use these new programs and devices effectively and leads us to believe that present computer technology may still be somewhat overwhelming for many adult users seeking to level the playing field with the sighted community. Our findings also reveal the need for training of professionals and tech support to ensure quality service. Getting feedback from users when developing new technology is also part of the accessibility and usability solution for actual and future technological development. Unfortunately, comments from our sample of users convey how little the sighted community is properly trained to support users of screen reading technology when accessibility, usability, or technical issues occur.

Of course, this study is not without limitations. Based on the data about their computer technology use, the 56 respondents are unlikely to be a representative sample of blind persons in general. More research is needed to deepen our understanding of issues faced by different language groups, and the constants or variances between different types of screen readers. Future research could also analyze different devices based on specific tasks to be accomplished. 


\section{Implications for Practitioners and Families}

While it is difficult to fix all the problems that can potentially hinder user interaction, the most frequently cited problem that blind persons cannot solve without outside help remains inaccessible content. If industrial content developers could systematically apply the Web Content Accessibility Guidelines (WCAG) and crucial standards such as providing text alternatives for non-text content, captions, alternatives for multimedia content, and making PDFs accessible, this would potentially solve the most common accessibility issues that blind persons cannot solve without sighted help. For usability issues, adding contextual support online could also benefit users on complex websites, such as in online shopping. Also, tech support of large companies must be better trained to properly assist blind customers using screen reading software.

While considerable progress has been made in user-led design, there is still some way to go in renewing the human side of eaccessibility, meaning expert-providers and disabled users need to work together to give a louder and more collective voice to eaccessibility. The legislative work in the last 25 years in the United States is a model to follow worldwide, since it is making e-accessibility a reality on computer-generated platforms such as Apple products, eBay, Ticketmaster, and Amazon with agreements reached after years of strong advocacy and collaboration.

In the future, videos of blind power users could be produced to demonstrate simple strategies used to manage tricky applications and environment.

\section{Conclusion}

This study investigated the technical difficulties and technical help that blind adults experienced when interacting with computers and computing applications. The findings indicate that the most cited technical problems related to upgrading or switching to new operating systems and inaccessible content, plus problems dealing with computer support in large companies.

This research study adds to the scientific knowledge base where very little literature exists about the types of problems that blind adults experience when using computing technology. This data helps to elucidate the issues that these moderate to expert blind adult users experience when dealing with computer technology and tech support. It allows us to assess the strategies used to solve issues and the particular types of problems that may or may not require outside assistance. The resulting data indicates that the respondents were able to deal with the majority of their technical problems. Yet, there still is a significant set of issues that require assistance, which would need to be communicated to professionals interested in e-inclusion. Further investigation on the impact of these issues on wellbeing, employment, and self-esteem is of interest to us for future research. It is also imperative to consider new ways to simplify the configuration when switching or upgrading to new operating systems and to ensure that contextual help for screen reader users is available on complex websites. If we are to support the claim that computer technology has become a democratizing tool, then it is imperative to continue investigating how blind adults are keeping pace with the rapid developments in the technological domain.

Table 1: Distribution of Participant Demographics, Types of Systems, and Devices Used

\begin{tabular}{|l|l|l|}
\hline Category & Total Number \\
\hline Gender & 25 \\
\hline Male & 31 \\
\hline Female & 31 \\
\hline & \\
\hline
\end{tabular}

\section{Level of Blindness}

\begin{tabular}{l|l}
\hline At most light perception & 46 \\
\hline Functionally blind & 10 \\
\hline &
\end{tabular}




\begin{tabular}{|l|l|}
\hline $18-25$ years old & 13 \\
\hline $26-35$ years old & 9 \\
\hline $36-45$ years old & 15 \\
\hline $46-65$ years old & 15 \\
\hline 66 plus years old & 4 \\
\hline &
\end{tabular}

\section{Type of Device}

\begin{tabular}{l|l}
\hline Desktop & 53 \\
\hline Laptop & 51 \\
\hline Tablet & 10 \\
\hline Mobile phone & 35 \\
\hline & \\
\hline
\end{tabular}

\section{Types of Operating System Used}

\begin{tabular}{|l|l|}
\hline Windows & 52 \\
\hline Mac & 15 \\
\hline iOS & 31 \\
\hline Android & 7 \\
\hline & \\
\hline
\end{tabular}

Number of Years Using a Laptop

\begin{tabular}{|l|l|}
\hline $15+$ years & 14 \\
\hline 6 to 14 years & 25 \\
\hline 5 or less years & 12 \\
\hline & \\
\hline
\end{tabular}




\section{Number of Years Using a Desktop}

\begin{tabular}{l|l}
\hline $15+$ years & 41 \\
\hline 6 to 14 years & 10 \\
\hline 5 or less years & 2 \\
\hline & \\
\hline
\end{tabular}

Number of Years Using a Tablet

\begin{tabular}{l|l}
\hline $5+$ years & 0 \\
\hline 3 to 5 years & 1 \\
\hline 2 or less years & 9 \\
\hline & \\
\hline
\end{tabular}

Number of Years Using a Mobile Phone

\begin{tabular}{|l|l}
\hline $5+$ years & 12 \\
\hline 3 to 5 years & 13 \\
\hline 2 or less years & 10 \\
\hline
\end{tabular}

Note: Participants could choose multiple answers.

Figure 1: Types of Technologies Preferred by Blind Users

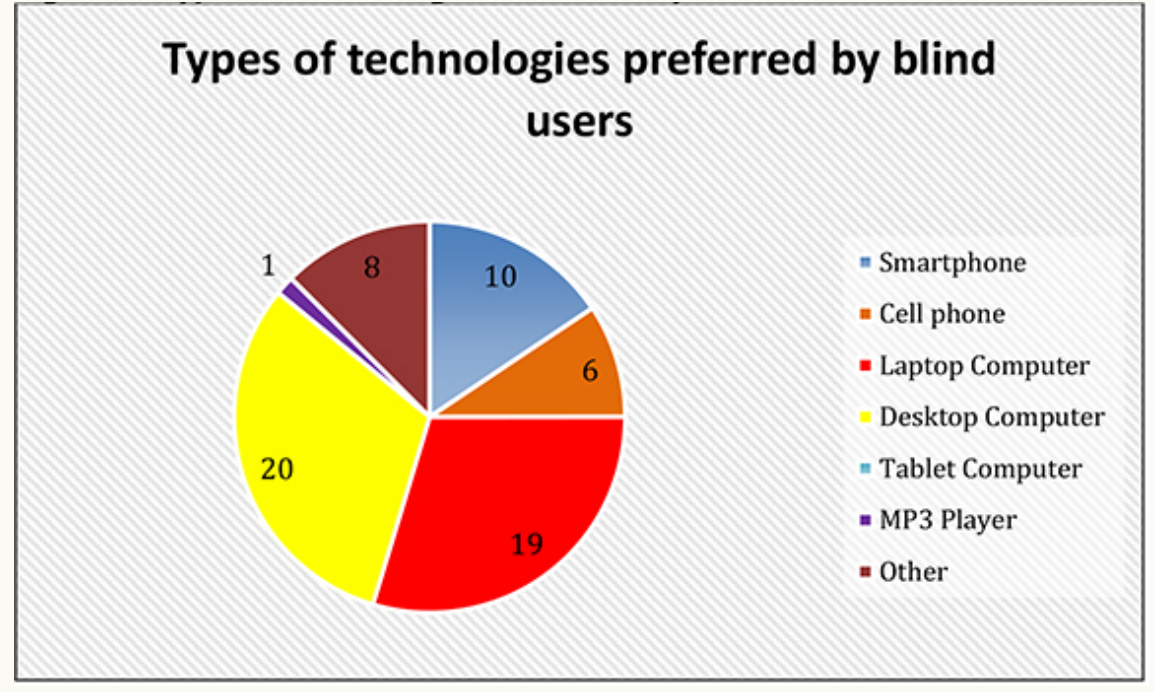

This pie-chart indicates respondents preferred technology in this order: 
1. Desktop computers with 20 respondents: $35.7 \%$

2. Laptops with 19 respondents: $33.9 \%$

3. iPhones with 10 respondents: $18.7 \%$

4. Others with 8 respondants: $14.3 \%$

5. Other Cell phone with 6 respondants: $10.7 \%$

6. MP3 Player with one respondant: $1.7 \%$

7. No participants indicated a preference for using a tablet.

Table 2. Distribution of Participants According to Their Help-seeking Behavior in Case of Training Needs.

\begin{tabular}{|l|l|l|}
\hline Behavior & English-speaking American & French Canadian \\
\hline $\begin{array}{l}\text { Mostly self-trained, with online } \\
\text { listserv, or virtual training tools }\end{array}$ & $29(67.4 \%)$ & $2(12.5 \%)$ \\
\hline $\begin{array}{l}\text { Mostly supported by tech company } \\
\text { trainers or rehab professionals }\end{array}$ & $11(27.5 \%)$ & $14(81.5 \%)$ \\
\hline
\end{tabular}

Note: Chi square $(1, n=56)=16.65, p<.05$

\section{Appendix A: Other Issues Regarding Tech Support}

Please describe other issues that are important regarding technical support for blind and low vision people that were not covered by this questionnaire:

\section{Open-Ended Responses}

All responses have been reproduced exactly as they were submitted by the survey respondents.

\section{English-speaking Americans}

It is important that if you are calling a company who is not familiar with working with blind people that the reps learn how to describe by key stroekes the products they are supporting. Most blind people do not use a mouse and they are not able to see the icons on the desktop. The company also needs to know enough about the two major screen readers so that they understand what is heard when clicking on an icon on the screen.

Manuals be available in accessible format when possible.

It is also easier when you call for technical support if you can fully understand the person assisting you because of their ability to speak English.

I think this is a problem with newer users of technology, but a tech support person needs to find multiple ways to explain the same problem. What's a simple explanation for someone who can see the screen can be incomprehensible for someone who is blind and new to all of this.

Sometimes it is just a matter of time to figure out problems and learn about assistive technology and I do not have all that extra time.

Companies that don't want to fool with us should hire a sub-contractor who knows something to do it for them instead of just giving the run-around or saying maybe the next version of their product will be better.

Familiarity with screen readers and Voiceover gestures. Telling a person who is blind where something is on the screen is pointless unless they are using a touch screen. Knowing how to manipulate the screen reader or use the different gestures for Voiceover is good. Being patient is also good.

The obvious one - when calling tech support for a non-blindness company/website, they rarely understand that I can't see the screen and they don't know how a screen reader works. So their instructions are all visual and it's hard to 'speak the same language' as them.

More people at Apple should be trained on how to properly use Voice Over as opposed to one go to person.

I would really be interested for you to ask questions of those of us who tend to be the techies in our families or friend circles. That's probably why I've answered so many of the questions in the way that I have.

The biggest issue is that technicians are absolutely clueless about using a system without a mouse and don't understand that color and screen-position of boxes etc is different for screen-reader users than for those looking at a screen. "just click on the orange box in the upper righthand corner" always happens at some level or other unless the technician actually has some idea of how addaptive technology works. 
Technical support staff at large companies should be trained on the basics of screen-reader use and function so they can communicate more effectively with blind customers.

Financial cost of hardware and software

When calling tech support most technician's have no knowledge of screen readers and will point the finger at the screen reader as the problem.

Counselers for commition for the blind and visual services have had NO training on what it is like to be blind.

It would be helpful if technology manufacturers had familiarity with programs used by people who are blind so their help desk representatives could provide keyboard shortcuts as opposed to always instructing people to click with the mouse.

People who are not familiar with assistive technology may find it hard to give instructions to blind users over the phone. The best thing to do is communicate that fact to the person helping them.

need hands on guidance. need to break technical language down into understandable terms, not everyone is a computer programmer or has the knowledge base of an expert. feel welcomeing as sometimes you are made to feel like a bother or ignorant by asking what others may view as a simple question.

The primary area of support is in the area of household electronic products-phones, television related equipment, etc. Many of these devices are far more difficult to use, beyond basic features, and awareness of access issues by manufacturers is very low.

You faile to take into account the existance of the various types of assistive technology used in varying combinations which may or may not be available for adapting computers, laptops and tablets. You do not take into account the use of stand alone assistive devices such as a Note Taker. Most I T and help desk personnel have no idea how to work with someone using a screen reader or other assistive gear. Written help nearly always gives mouse commands and never keyboard alternatives. I could go on.

\section{French Canadian}

It would be nice to have tutorials or self guided resources to solve problems.

When you are talking to a help agent on the phone, it is hard to have them understand that I can't see the screen.

Lack of screen reader accessibility knowledge on the part of tech support providers in certain situations discourages me from seeking out mainstream tech support if I believe that lack of screen reader compatibility is an issue.

I think more people need to be trained in low vision/blindess products. Especially website builders. It's getting better, but some sites are so inaccessible I cannot use it affectively.

The biggest issue for me is insufficient training at a cost I can afford and in some instances the training just doesn't exist.

As I have mentioned in other places, it is nice when technical support people have some understanding of adaptive technology.

For those of us who live rurally, learning a new operating system is difficult and expensive. My trials with a tablet have proven unsuccessful because there are no blind persons or trainers nearby who are familiar with the Android operating system's use by blind people. I have thought about returning to a Macintosh computer but there is no training available locally except itinerant trainers whom I must pay for travelling as well as training and travel is usually 100 miles or more. This becomes prohibitively expensive if it is available at all.

I think it is difficult to explain to most sighted folks how a screen reader works. Example, spatial orientation such as "just to the left of" doesn't compute for a blind person. The screen reader likely rearranges that relational information.

It is extremely helpful when the person assisting me has at least a basic knowledge of how a blind person uses a computer. If they lack this knowledge, it helps if they are an adept listener who picks up quickly on my explanations. When somebody starts telling me to press the blue button at the top left of the window after I have explained I do not see, this is less than useful.

education of tech assist persons on adaptive products for the blind is needed

When calling tech support, it is difficult to get the technician to understand that they cannot tell a blind person to click on the icon on the upper right, or anywhere else for that matter, but that they need to know how to communicate how to access something that does not involve the mouse.

Getting to start using a product like Vinux is not advertised and the upside to why it is better than what the user is currently using is very hard to find.

While I haven't experienced this myself, I have been told that many people, upon calling mainstream technical support, find that the person on the other end of the line has no idea how to solve problems in ways that don't involve sight.

It is critical that those providing such support have at least a basic understanding of the adaptive tech that is in use. I have had to clean up too many messes resulting from "knowledgable" friends helping a client but not grasping that what was just done breaks a critical aspect of the client's adaptive tech.

\section{References}


Abner, G. H., \& Lahm, E. A. (2002). Implementation of assistive technology with students who are visually impaired: Teachers' readiness. Journal of Visual Impairment \& Blindness, 96(2), 98-105.

Bastien, C. (2015, March). User Experience Evaluation: An essential step. Paper presented at the 8th European e-Accessibility Forum User-driven e-Accessibility, Paris, France. Retrieved from http://inova.snv.jussieu.fr/evenements/colloques/colloques/article.php? $\underline{c}=82 \& \mid=e n \& a=441 \#$ contenu article

Lazar, J. (2009). Making CAPTCHA more accessible for the blind. Braille Monitor, 52(1). Retrieved from https://nfb.org/images/nfb/publications/bm/bm09/bm0901/bm090108.htm

Percival, J. (2012). Demonstrating daily living devices to older people with sight loss: A programme evaluation with implications for good practice and policy development. British Journal of Visual Impairment, 30(2), 79-90. doi:10.1177/0264619612442069

Petty, R. E. (2005). Technology access in the workplace and higher education for persons with visual impairments: An examination of barriers and discussion of solutions. Independent Living Research Utilization at TIRR, Houston, Texas. Retrieved from http://www.ilru.org/sites/default/files/Technology_Access Visual Impairments.pdf

Smith, D. W., Kelly, S. M., \& Kapperman, G. (2011). Assistive technology for students with visual impairments: A position paper of the division on visual impairments. Retrieved from http://community.cec.sped.org/dvi/resourcesportal/positionpapers

Zhou, L., Parker, A. T., Smith, D., \& Griffin-Shirley, N. (2011). Assistive technology for students with visual impairments: Challenges and needs in teachers' preparation programs and practice. Journal of Visual Impairment \& Blindness, 105(4), 197-210.

The Journal of Blindness Innovation and Research is copyright (c) 2017 to the National Federation of the Blind. 